Before referring to these later effects of trauma, mention may be made of an intermediate group of cases in which initial symptoms are absent or slight and grave symptoms come on a few days or weeks after the injury, having the character and course of a subacute myelitis.

Thus Lohrisch ${ }^{3}$ reports the case of a man, aged 58 , who, after a fall on his back, suffered for a fortnight from violent pains on attempting to move his legs. During the next fortnight the pains were less severe, but on the twenty-ninth day after the accident there quickly developed a complete motor and sensory paralysis of both legs together with paralysis of the bladder. At the necropsy a softened focus was found in the dorsal region.

Gowers, 4 too, mentions the case of a lady who was severely shaken in a railway collision. Immediately after the accident ' she seemed to have suffered no injury, but in a few days paraplegia developed, and from its consequences she died six weeks after the accident. Throughout the dorsal portion of the cord indications of a subacute myelitis were fouud, chiefly in the white columns.

These cases bridge over the interval between the earl severe changes, whether molecular or coarse, and later changes, more or less degenerative in character, which underlie the symptoms of locomotor ataxia, disseminated sclerosis, chronic mvelitis, and other slowly progressive diseases of the cord. The symptoms of these disorders may not appear until several months aiter an accident, and their dependence on morbid changes produced by concussion can only be inferred after the exclusion of other causes.

On going over cases of spinal disease which have been under my care during the last fifteen years, the collection of hospital cases having been kindly summarized by a former house physician, Dr. Charles O'Neill, I find that a relation to trauma can be traced more frequently in disseminated sclerosis than in locomotor ataxia. The following are two examples :

\section{CASE VI,}

A woman, aged 30 , previously hesithy, fell through a trap door into a cellar, a distance of $5 \mathrm{ft}$., and dropped on to a stone slab in a sitting posture.- A few days later she complained of numbness on the left side of her face and back. In about flve months she began to have a difficulty in walking; this increased, and spastic paraplegia slowly developed. This was followed by nystagmus and weakness of the arms. When seen at a later period the symptoms were unmistakably those of disseminated sclerosis.

Case vir.

A men, aged 27, fell over a banister on to his back. He was unconsclous for four days. After a week he went to work, but could not stand properly. The weakness in the legs increased, and when examined two years after the accident he had well-marked signs of disseminated sclerosis.

Much has been written on the subject of traumatic tabes and in a few cases it seems probable that concussion has started the changes in the posterior root fibres. A patient of my own stated that his ailment dated from a fall three months before he came to see me. He.slipped down some thirty stone steps, jogging the lower part of his back on each step. He walked home and went to bed. Two days later he began to stagger, and had pains in the soles of his feet. On examination the patient showed a moderate degree of ataxia. The knee-jerks were absent. The pupils were unequal, and did not react to light. Micturition was delayed.

Lichte, ${ }^{5}$ who has written a thesis on traumatic tabes, gives a full account of a case in which a severe sprain of the left arm was followed in a few months bv signs of tabes. Syphilis and other possible causes could be excluded

These cases, however, are exceptional, and a study of the literature, as well as one's own experience, leads to the conclusion that although concussion may initiate the development of characteristic signs of tabes it rarely acts as the sole cause of this.disease. Frequently it will be found on careful inquiry that the tabetic patient was not quite free from symptoms before the accident, No doubt trauma often cooperates with other causes, especially syphilis, and tends to light up disease which was pre-existent, or for which the ground was duly prepared.

IfJury to the Periphery of the Body.

Passing now to the periphery I will consider only the question whether disease of the spinal cord may result from injury to the peripheral nerves. A good starting point, because the condition is well known, is arthritic muscular atrophy. Articular inflammation, whether produced by injury or disease, is almost invariably followed by rapid wasting of the muscles that move the joint, the extensor muscles being predominantly affected. Thus, if the shoulder is injured the deltoid chiefly wastes ; if the elbow the triceps ; if the hip the glutei ; if the knee the quadriceps. The atrophy comes on quickly so that in case, for example, of injury to the knee a difference of an inch between the girth of the thighs might be noticed at the end of a week or ten days. As to the explanation of this condition, the evidence both experimental and pathological gives support to the reflex theory held by Paget, Vulpian, and Charcot, which is that the muscular atrophy depends on a damaged nutrition of the motor cells of the cord, this derangement being the effect of morbid impulses from the irritated nerves of the injured joint. The correctness of this view is practically proved by the experiments of Raymond, who found that a previous division of the posterior spinal roots prevented the wasting of the muscles.

Further light has been thrown on the subject by the investigations of a former Manchester student, Dr. Warrington, who has shown that actual changes in the motor cells may be detected after lesions of the posterior roots.

There can indeed be no doubt that healthy sensory impulses are essential for the proper nutrition of motor neurons, and that morbid sensory impulses will lead to detectable disease. Do the morbid changes pass away when the cause-say articular inflammation-is removed, or do they ever progress and lead to atrophy and destruction of the motor cells ? In other words, may progressive muscular atrophy result from peripheral irritation? The answer is that occasionally the muscular atrophy resulting from arthritis becomes permanent, and that many cases of progressive muscular atrophy appear to originate from a severe sprain or other injury to a limb. I have notes of several instances of this relation. A few years ago I showed a patient at a meeting of this Society in which the sequence was striking.

CASE VIII

The patient, a powerfully built policeman, aged ${ }_{3} 6$ years, was admitted in the infirmary with characteristic signs of progressive muscular atrophy, the muscles of the right arm being principally affected. He attributed the onset of his ailment to a violent struggle with a maniaca person in which he strained the upper part of the right arm. About a person in monted when he came under my care twenty months after the injury.

The boundary line between cases of arthritic muscular atrophy and chronic anterior poliomyelitis is not always easy to define, and the differential diagnosis may present great difficulties.

In conclusion, I would draw attention to the need for further study of the after-effects of trauma, and especially as regards their prognosis. Traumatic neurasthenia and hysteria have received ample consideration, but there is room, I think, for a fuller recognition of the prolonged and sometimes permanent incapacity for work that may result from concussion of the head or the body.

Adequate recognition of the possibility of disease leads to increased care in preventive treatment, and to mention only one thing, I believe that the simple prescription of a longer rest in bed after injury than is usually obtained in hospital practice would do much to reduce the number of sufferers from chronic diseases of the nervous sy stem.

REFERENCES.

1 Page, Concussion of the Brain, Brain, 1902. 2 Crisp English, Hunterian Lectures. xg04. 3 Lohrisch, Zur path. Anatomie der posttraumatischen Diseases of the Nervous System vol. i. 5 Lichte, Traumatische Tabes, 1903.

THE EXAMINATION OF THE TISSUES. OF THE CASE OF SLEEPING SICKNESS IN A EUROPEAN.

[With Special Prate.]
GEORGE (: LOW,
and
F. WALKER MOTT, M.A., M.B., M.D., F.R S..

Medical Superintendent and Tutor, London School of Tropica] Physicían Charing Cross Hospital; Pathologist, Londom Medicine. County Asylums.

IN October, I902, Sir Patrick Manson was consulted by the wife of a missionary from the Congo suffering from chronicpyrexia and enlargement of the spleen. After an examination he came to the conclusion that the disease was probably trypanosomiasis, and sent the patient to the wards of the London School of Tropical Medicine, where Dr. Daniels confirmed the diagnosis by finding trypanosomes in the blood. A full account of the case was published in the British Medical Journal of May 3oth, 1903, by Manson and Daniels, and the termination and results were described later by Manson in the same journal, December $5^{\text {th, }} 1903$.

The symptoms that were present when the patient was 


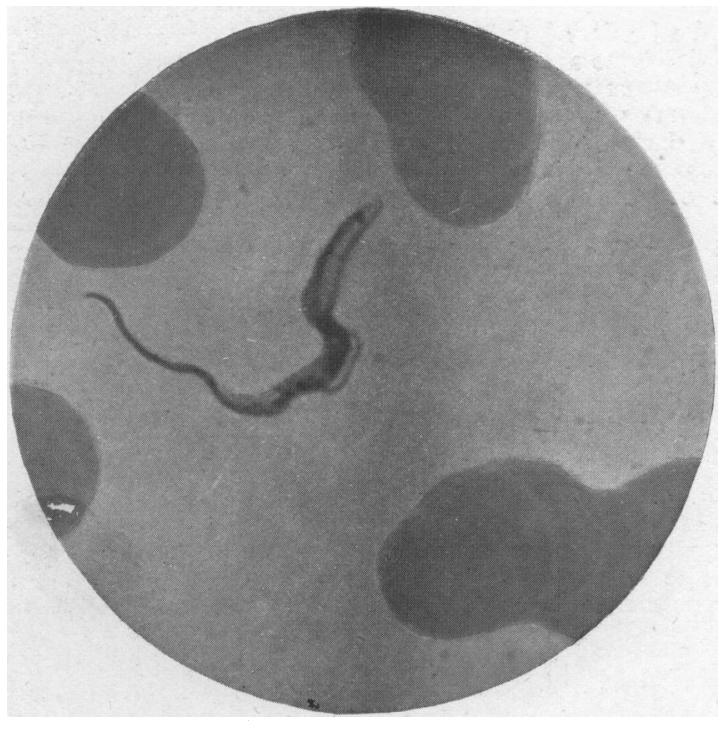

Fig. r.-Film preparation of blood with trypanosomes. Magnification 2,00o diameters.

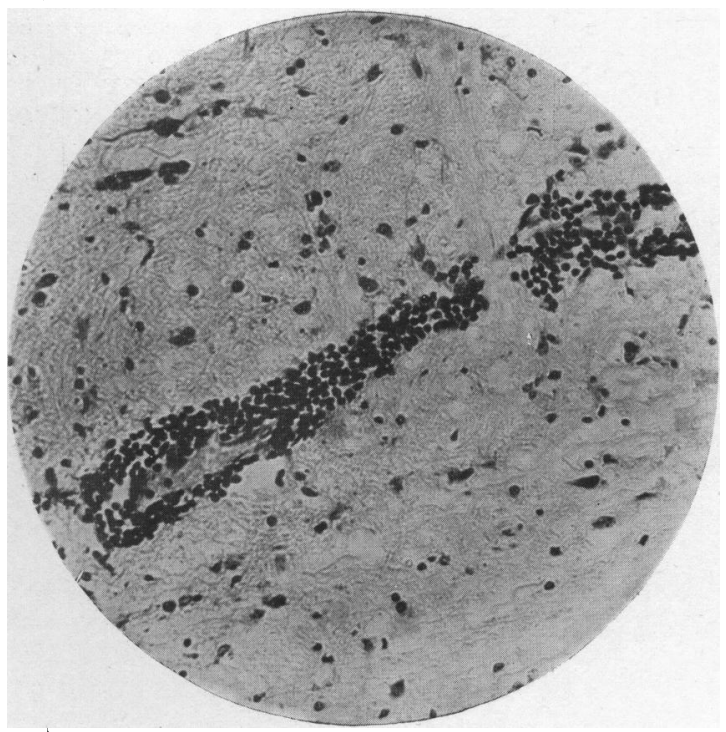

Fig. 3.-Small vein of medulla oblongata showing the surrounding accumulation of mononuclear leucocytes. Magnification 175 diameters.

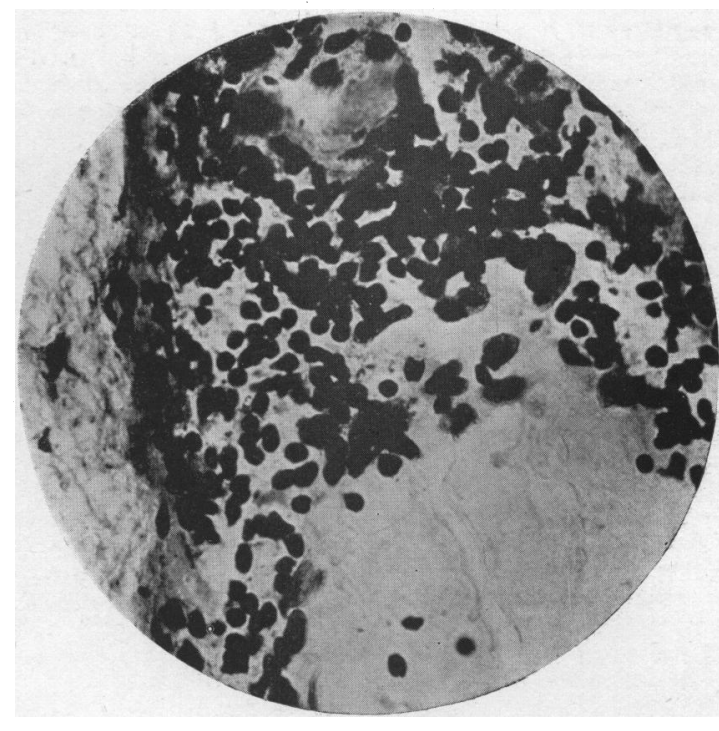

Fig. 2.-Perivascular infiltration of mononuclear leucocytes-cerebellum. A number of small bodies (not diplococci or disintegrated cells) possibly degenerated trypanosomes. Magnification 500 diameters.

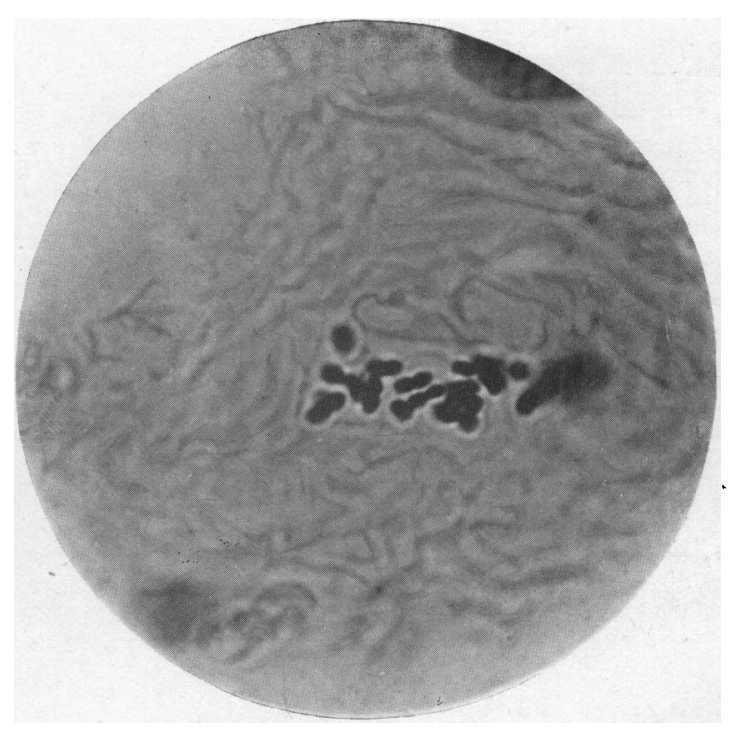

Fig. 4.-Diplococci in brain. Magnification 2,500 diameters. 


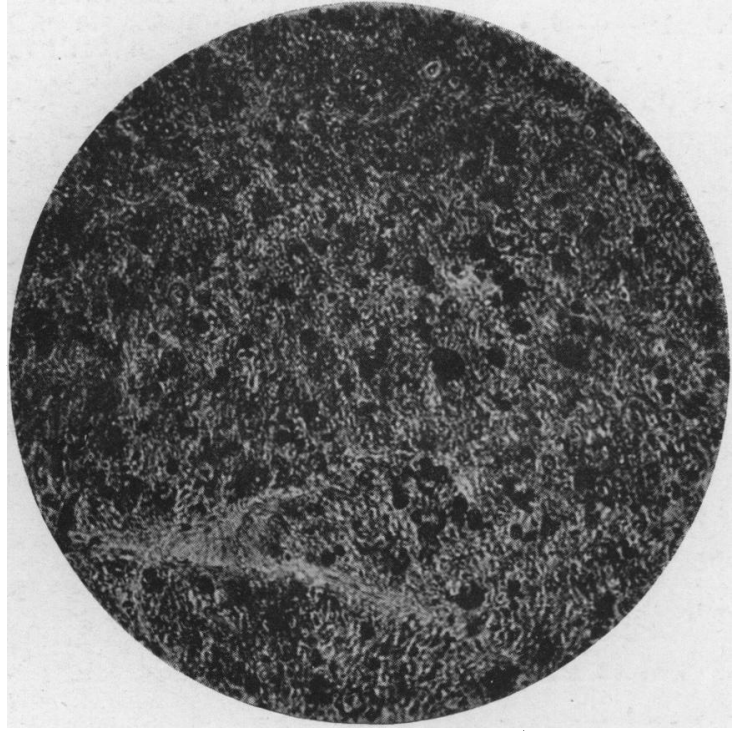

Fig 5.-Degenerated fibres of spinal cord by Marchi's method. Magnification 200 diameters.

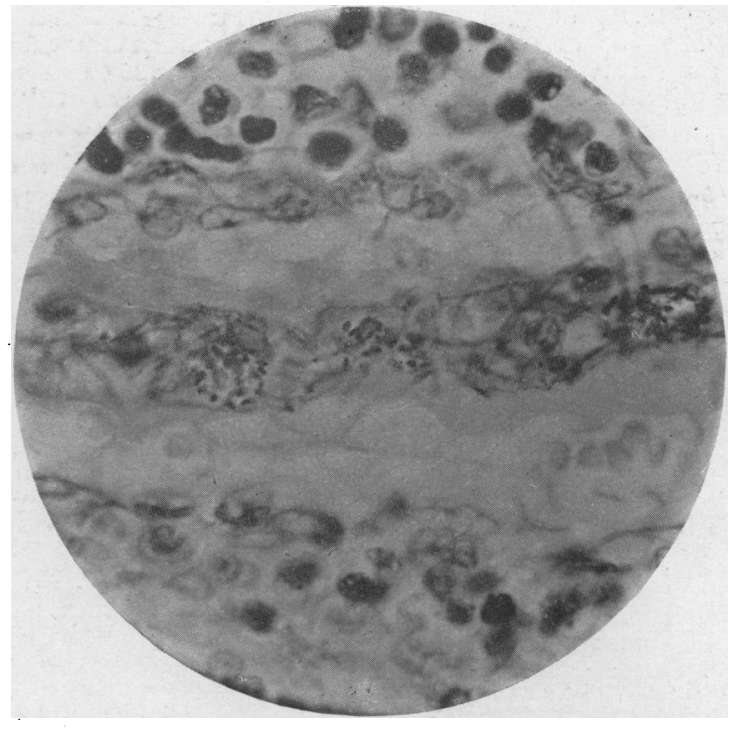

Fig. 6.-Diplococci in a lymph channel of lymphatic gland Magnification 800 diameters.

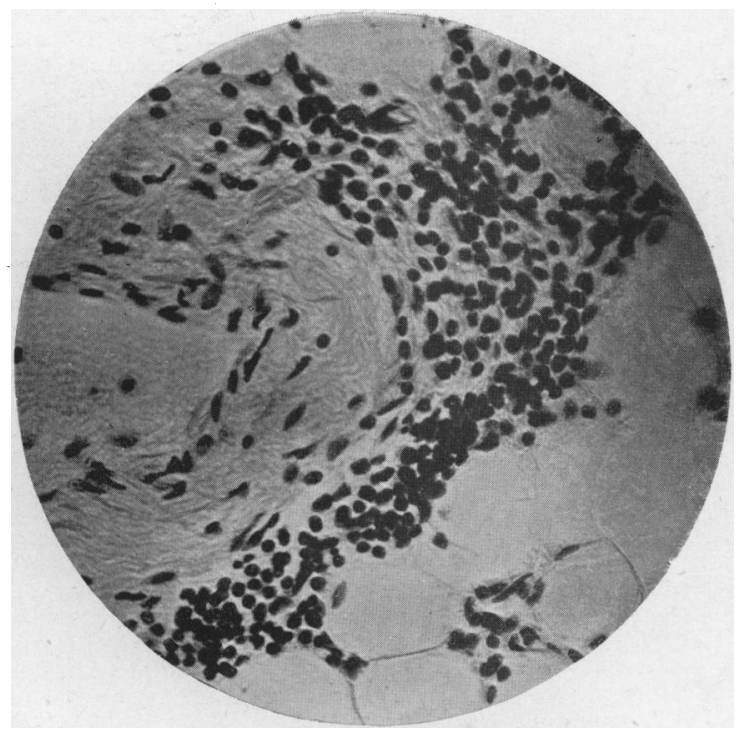

Fig. 7.-Perivascular mononuclear infiltration-epicardium. Magnification 400 diameters.

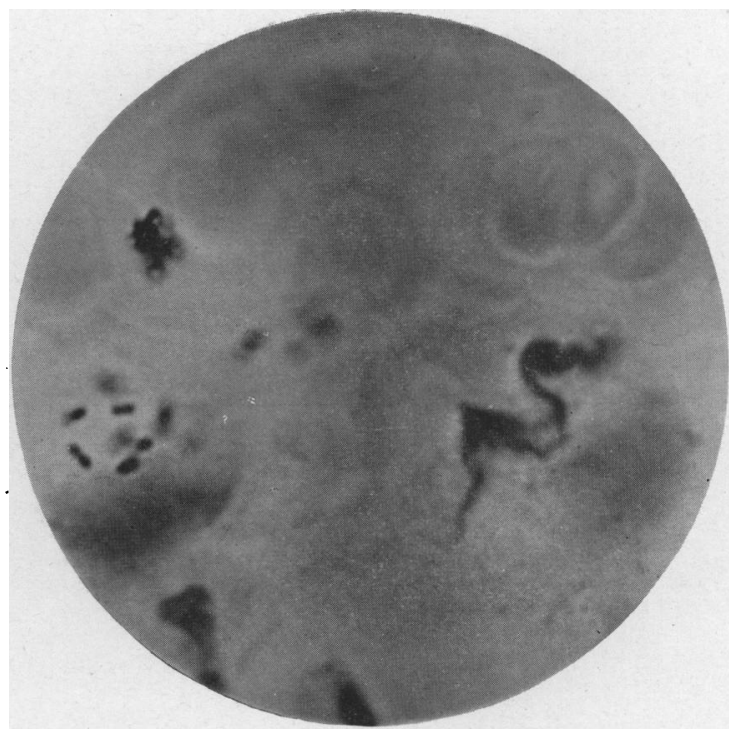

Fig. 8.-Diplococci and dead trypanosoma in a small haemorrhage. of myocardium. Magnification 2,00o diameters. 
under observation in hospital were chronic irregular pyrexia, emaciation, enlargement of the spleen, a peculiar erythema. tous condition of the skin, and the presence of trypanosomes in small numbers in the peripheral blood. At that time there were no signs of tremors, drowsiness, or other nervous symptoms associated with sleeping sickness.

After leaving hospital a change to the seaside was advised, and the summer was chiefly spent at Cromer. In October, 1903, the patient returned to her home in Bristol, and for the first time about the middle of that month a marked tendency to drowsiness appeared. With the exception of some twitchings at the left angle of the mouth there were no other evidences of tremors. Emaciation, however, had now become extreme, and small hard glands were palpable in the posterior triangles of the neck. " She gradually became more drowsy; in the last days of life the sphincters relaxed and she died in a state of coma.

\section{NeCropsy.}

We are indebted to Dr. Neill, University College, Bristol, who made a necropsy two and a-half hours after death, for the following notes of the macroscopic conditions :

\section{External Appearances.}

Skin 'yellowish over body, rough and dry on the upper arms, thighs, and abdomen: sclerotics tinged yellow, pubic and axillary hair very scanty. - Great general emaciation, mammary glands small and sharply outlined. Early bedsores over sacrum, posterior ends of fliac crests, very early over right trochanter; skin tightly drawn over facial bones. No obvious oedema, but slight pitting over tibiae on firm pressure. Rigor mortis present in jaw muscles only.

Head.

(Brain examined first.) Calvarium unchanged, dura mater normal ; a quantity of blood and cerebro-spinal fluid escaped on removing the brain, and the infundibulum was accidentally opened. Pia mater finely injected, and veins distended with blood. Injection difficult to remove with finger pressure, more marked over the occipital lobes. No glazing of surface. Convolutions sharply rounded, and sulci wider than normal, this being very marked on the right side. Larger sulci flled with clear fluid, and the superjacent membrane slightly hazy. Cirele of willis normal. No atheroma. Excess of fluid in basal lymph eistern, with injection over medulla and pons, otherwise nothing abnormal at base. No adhesions in longitudinal fissure. Corpus callosum and fornix quite firm. Lateral ventricles dilated and contained excess of fluid. Ependyma injected, but brain tissue not softened. No abnormality discovered macroscopically in centra, basal ganglia, crura, cerebellum, pons, or medulla. No abnormal fullness of cerebral vessels. No thickening of 'meninges over anterior end of upper surface of vermis; no obstruction of veins of Galen.

Spinal cord not opened, but about 2 in. of cervical cord removed. No obvious thickening of membranes. No macroscopic changes on section.

Thorax.

Small remains of thymus, and one or two small, deep-red, lymph glands found under manubrium sterni. Lungs: Right, a tew thin bands of old adhesions laterally and above upper lobe. Lower lobe firm to the touch and covered with fine recent pleurisy. On section some fibrous pigmented thickening at extreme apex of upper lobe, probably healed tubercle. Rest of lobe showed oedema. Middle lobe normal. Lower lobe: Bronchioles congested, substance pale red, uniformly firm airless, and non-crepitant, granular on section. Left, adnesions and fibrous tissue at apex similar to right, but less extensive. Rest of lung and pleura normal.

Pericardium uniformly adherent, but stripped fairly easily except over roots of great vessels. Heart cavities a little dilated, and the auriculo-ventricular orifices admitted the fingers more easily than normally. Mitral and tricuspid valves showed chronic fibrous thickening, with one or two small raised nodules of old fibrous tissue. No recent endocarditis. Semilunar valves and coronary arteries normal. Minute patches of atheroma in a orta. Heart muscle normal colour, no fatty or patches of atheroma in aorta. Heart muscle normal colour, no fatty or clotted rapidly on being shed.

Abdomen.

No fluid in abdominal cavity. Peritoneum glazed over upper part of small intestine. A patch of inflammation on surface of jejunum. Intestines not opened. Spleen occupied upper left quadrant of abdomen, reaching to within an inch of the umbilical level, and crossing the middle line by a fingerbreadth. Uncovered, except by the omentum, at the anterior margin. The spleen was uniformly enlarged, and the margins rounded, the hollow at the hilum being filled out. Capsule somewhat thickened, but no perisplenitis except at the upper end where the organ was suspended by a thick band of flbrous tissue as thick as the little finger. On section the substance was firm, but not markedly so. Surface pale, opaque; trabeculae thickened : Malpighian bodies clearly seen and sparsely distributed.

Liver.-Enlarged; firmer than normal. No perihepatitis, although a little recent lymph at right anterior margin. Margins rounded. Surface smooth, but, on closer inspection, fine no:tular arrangement visible. Firm on section; colour browner and darker than normal showed condition of cirrhosis.

Kidneys.-Tougher than normal ; cortex and medulla almost the same colour; former diminished. Capsule stripped with a little difficulty, leaving a granular surface. Kidneys smaller than normal.

Suprarenals. - Healthy on section.

Lumbar Lymphatic Gland8.-Enlarged, intense red colour.

Uterus.-Nulliparous. Small fibroids.

Bladder and appendages healthy.

Pelvis.-Normal.

Bme Marrow.

Taken from left humerus. Marrow in cancellous spaces pale red with some fat, in shaft dark red, barely keeping together. No yellow fat een.

Blood films were prepared by Dr. Symes from the heart and spleen. Portions of the different organs were sent to us in Müller's solution, formalin, and in alcohol for microscopic examination, and arrived on the following day.

\section{The Blood.}

Unfortunately the spinal fluid was not examined for trypanosomes, but blood films taken a week before death, and those taken by Dr. Symes from the heart and spleen at the necropsy, though carefully examined, have shown no trypanosomes. The characteristics of the blood are similar to those of the peripheral blood described in the former report of the case, namely, some changes in the size of the red corpuscles, nucleated red corpuscles, and an increase (relative) of the large mononuclear leucocytes. In addition, however, all the films show very clearly the presence of large numbers of diplococci, these being more numerous in the heart than in the spleen.

\section{Microscopic Examination of the Tissues.}

The sections were for the most part cut in paraffin from tissues hardened in formalin or alcohol, but those stained by Marchi method were prepared from those hardened in Müller's fluid and the sections cut in celloidin. The sections cut in paraffin were stained with carbol-thionin and erythrosine, Unna's polychrome, or logwood and eosin. Sections of the brain were made from various parts of the cortex, the cerebellum, the pong, and the medulla. The spinal cord was also examined. The posterior spinal ganglia and the roots were unfortunately not sent.

Description of the Changes.-I. Central Nervous System.

Membranes. - The pia-arachnoid membrane showed an infiltration, with large and small celled mononuclear leucocytes of a not very marked character in the sections of the cortex, but much more marked in those regions where there is a larger amount of cerebro-spinal fluid, for example, the cerebellum and the medulla.

Vessels.-In the cortex the small vessels, especially the capillaries, frequently show swelling and some proliferation of the endothelial nuclei; this, however, is more marked in some places than others. The blood contained in the vessels exhibits usually an excess of mononuclear leucocytes; in some places groups of polynuclears are seen. Around the vessels are found the characteristic mononuclear infiltration first described by one of us (F. W. M.), and since confirmed as pathognomonic of this disease. This infiltration is much more marked in some portions of the cortex than others, but it is very well marked in the cerebellum and the medulla oblongata. (Figs. 2 and 3.) The vessels of the medulla and spinal cord are congested and in places, especially in the former, there are many recent capillary haemorrhages.

Nerve Cells.-The ganglion cells of the cortex and of the medulla, to a much less degree the spinal cord (in which latter the vascular changes are not marked), show acute changes, probably of the nature of coagulation necrosis. The cells are by no means all affected, nor to the same degree. There is chromatolysis, the Nissl granules being either indistinct, absent, or a dust-like powder, with absence of the granules on the dendrons, which may be broken off, or not exhibiting their normal forms. The change 3 , in our opinion, are mostly of recent origin, and like the capillary haemorrhages were due to the toxic conditions of the blood and cerebro-spinal fluid caused by the invasion of the diplococci to be described.

Glia Tissue.-The neuroglia cells in places show active proliferation.

Organisms. - No trypanosomes were found in the blood vessels or perivascular spaces of the central nervous system, although a large number of sections were examined. In the superficial cortex in some sections small foci of leucosytes, mononuclear, and polymorphonuclear were found. Several of these foci exhibited no diplococci, but curious granules of varying size, as if these may have been the cause of the inflammatory action. We would not venture to say that these were degenerated trypanosomes, but it is possible that they may have been such. Similar bodies were found amidst the cells in the perivascular spaccs of the medulla and cere- 
bellum (Fig. 3). The magnification, 500 diameters, is not sufficient to show these small round bodies distinctly. Under a high power, however, no differentiation of structure can be made out. It is therefore impossible at present to do more than record the fact of their existence.

In all parts of the central nervous system, as indeed in all the organs and tissues, diplococci (staining by Gram's method) could be found (Fig. 4).

By Marchi staining, numerous recently-degenerated fibres could be found in the medulla, spinal cord, and cortex (Fig. 5).

The microscopical examination, therefore, of the nervous system shows changes similar to those which have been met with by us in cases of the disease occurring in Africans.

Lymphatic Glands.-A large number of sections of these were examined, and by Gram's method every section showed large numbers of diplococci in foci or in the lymph channels and blood (Fig. 6).

Bone Marrow.- Showed diplococci and large numbers of large mononuclear leucocytes.

Lungs. - Congestion and pneumonia of the right lower lobe was found. Diplococei present.

Liver.-The cells exhibit fatty infiltration; there is some increase of fibrous tissue; the blood in the vessels shows haemolysis ; excess of mononuclear leucocytes and diplococci.

There is no malarial pigment.

Spleen.-Congestion of vessels, increase of fibrous stroma ; diplococci ; no malarial pigment.

Kidneys.-Vessels congested, no diplococei discovered; epithelium of uriniferous tubules exhibits first stage of coagulation necrosis, probably due to the toxic condition of the blood.

Heart.-Epicardium, a marked mononuclear infiltration around the vessels (Fig. 7). Early pericarditis: Myocardium, the muscle fibres exhibit brown atrophy and pigmentation, and there is a condition of recent haemorrhagic myocarditis due to infection; for in the haemorrhages are numerous colonies of diplococci, and in one section what appears to be a dead trypanosome (Fig. 8).

This is the only distinct evidence pointing to the existence of trypanosomes found in the large number of sections examined, but its form and size and its existence amid the blood corpuscles point undoubtedly to its nature, although it does not present the typical characters of the trypanosome seen in the film preparation (Fig. 1).

We have recorded the bare facts, and consider that this case is of especial interest, inasmuch as it shows that a European who has resided in a country where sleeping sickness exists, and who leaves that country with trypanosomes in the blood, may for a considerable time present no symptoms of sleeping sickness, and then develop them and die, the tissues showing changes similar to those met with in Africans. It is a strong argument in favour of the trypanosomes being the cause of the disease; but what part do the diplococci play? Have these appeared subsequent to the onset of the characteristic symptoms of the disease, and is there, therefore, a causal connexion, or is it merely a terminal infection?

We desire here to acknowledge the services of $\mathrm{Mr}$. Charles Geary, Assistant to the Pathological Laboratory, Claybury, for the excellent photomicrographs of his preparations of the tissues.

\section{ASYLUM DYSENTERY IN RELATION TO B. DYSENTERIAE}

By J. W. H. EYRE, M.D., F.R.S.EDIN., Bacteriologist, Guy's Hospital ; Lecturer on Bacteriology, Guy's Hospital

(From the Bacteriological Laboratories of Guy's Hospital.)

THE researches of Shiga, Kruse, Flexner, Strong, Rogers and others have resulted in the accumulation of a mass of evidence pointing to the correctness of the assumption that acute dysentery, wherever it occurs, is a disease due to the invasion of the human organism by a specific bacillusB. dysenteriae; or, perhaps, in view of the uncertainty existing at present with regard to the relative importance of the Flexner and Shiga types, it would be more exact to say some one or other member of that group of organisms typified by $\mathrm{B}$. dysenteriae. Flexner and other workers in this field, notably Vedder and Duval, have also demonstrated the presence of a similar bacillus in material obtained from cases of epidemic diarrhoea occurring in asylums for the insane in
America, thus supporting the contention of Mott, that the disease, is identical with the dysentery of tropical climes. So far as I am aware, however, the organism above referred to has not hitherto been iselated from cases of asylum dysentery in this country, and I therefore wish, to state briefly the positive results obtained from the bacteriological examination of material derived from such cases. My material was obtained from an epidemic of dysentery which occurred towards the end of last year in one of the London county asylums, and I now wish to express my thanks to Dr. Mottfor his kindness in placing the said material at'my disposal.

\section{CoURSe of THE EPIDEMIC.}

With the clinical aspect of the cases comprising this outbreak, and the means by which the disease was carried to wards other than that in which it first appeared, I am not at present concerned. Indeed many interesting points bearing on asylum dysentery and its bacteriology must be reserved for a detailed paper, which will appear in the forthcoming volume of the Archives of Neurology.

Briefly, the course of the epidemic was as follows: The male side of the asylum had been remarkably free from cases of diarrhoea and dysentery for some time prior to August 1903. On the 2oth of that month, however, two cases of dysentery were reported from a ward which I will call Ward A, and were followed by two more on the 24th; a fifth case occurred on the next day, and a sixth on the 28th of that month. No more patients were attacked in this ward, and within two months all six cases were convalescent.

Towards the end of September a similar outbreak, but of a more severe type, made its appearance in Ward B. Here eight patients were successively attacked, and of these three succumbed to the disease. In Ward $C$ three patients contracted the disease, of whom one died. Isolated cases, in ones and twos, cropped up also in other wards at varying intervals during the months of September and October to the total number of eight, three of these being fatal.

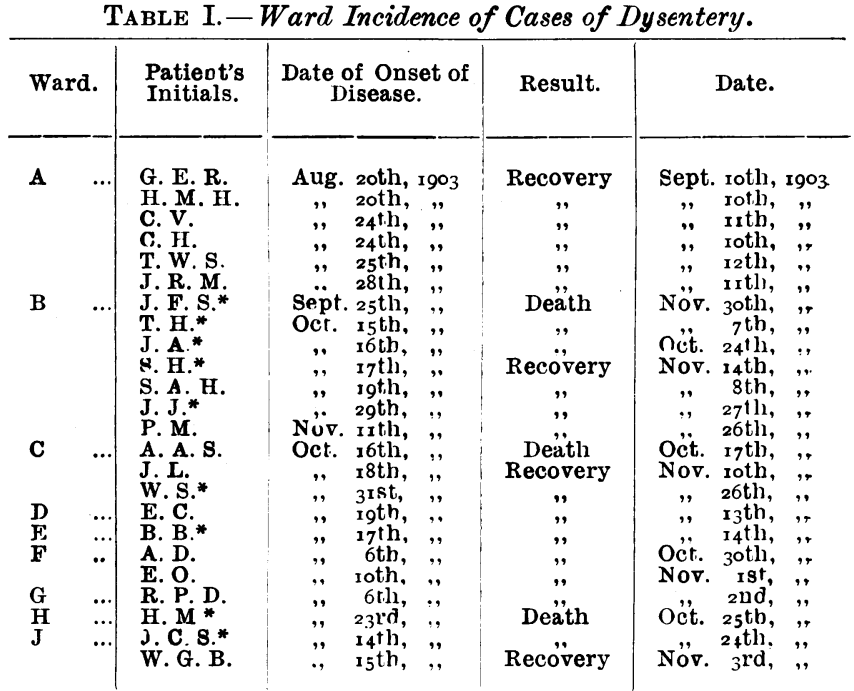

* Cases examined bacteriologically are indicated by an asterisk.

None of the cases in Ward A came under my observation, but of the remaining 18 cases I was supplied with specimens of the stools from 5 during life. Further specimens of faeces, scrapings of dysenteric ulcers, blood, and bile were collected at the post-mortem inspection from 5 of the 6 fatal cases (with all precautions against accidental contamination), and sent to me for examination.

Methods: Faeces,

The method adopted in the examination of the faecal material and scrapings from dysenteric ulcers was simple in the extreme, the work of differentiation and isolation being facilitated by the employment of a special nutrient mediuma modified form of the "lakmus-laktose-nutrose-agar" of Drigalski and Conradi.* A platinum loop, reserved for this particular purpose, and capable of holding about $2 \mathrm{mg}$. of faeces, was employed to emulsify three or more loopfuls of the specimen in $10 \mathrm{c.cm}$. of sterile nutrient broth. Three

* A simplified method of preparing and standardizing this medium formed the subject of a recent communication by the present writer to the Pathological Society, January roth, rgo: 